ARTí́CULO

\title{
Tratamiento de efluentes del cultivo de Seriola lalandi por sedimentación, filtración y absorción en diferentes tiempos de retención hidráulica
}

\author{
Treatment of effluents of the Seriola lalandi culture by sedimentation, filtration and \\ absorption processes at different hydraulic retention times
}

\section{Roberto Ramos $^{1^{*}}$ y Antonio Navarro ${ }^{1}$}

${ }^{1}$ Departamento de Ciencias Acuáticas y Ambientales, Facultad de Ciencias del Mar y de Recursos Biológicos, Universidad de Antofagasta, Av. Angamos 601, Antofagasta, Chile *Autor para correspondencia: roberto.ramos@uantof.cl

\begin{abstract}
On a laboratory scale, the removal efficiency of particulate material and dissolved nutrients contained in the effluent generated in the production of yellowtail Seriola lalandi was evaluated. Sedimentation processes, filtration by the oyster Crassostrea gigas and absorption by the macroalga Gracilaria chilensis in times of hydraulic retention (HRT) of 6, 12 and $24 \mathrm{~h}$ were applied. Conical cylinder tanks of $150 \mathrm{~L}$ capacity were used for the sedimentation process. In the filtration and absorption processes, acrylic conical cylinder tanks with $50 \mathrm{~L}$ capacity were used. All the treatments were in triplicate, except for the sedimentation process that used a single pond for each treatment. The highest removal of turbidity was registered at $12 \mathrm{~h}$ with a value of $73.44 \%$. The removal of total suspended solids (TSS) and total volatile solids (TVS) reached the highest efficiency at $24 \mathrm{~h}$ with 13.17 and $49.06 \%$, respectively. The removal of the nutrients does not present a high efficiency; however, the phosphate in $12 \mathrm{~h}$ was reduced in $82.23 \%$. In the filtration process, turbidity presents a high removal efficiency in all HRT evaluated, with the best result being $92.57 \%$ in $6 \mathrm{~h}$. For the TSS and TVS the removal results were 29.94 and $75.4 \%$, respectively. In this process the reduction of nitrogen nutrients was very low, even with negative values. However, for phosphate the greatest removal was recorded in $12 \mathrm{~h}$ with $88.32 \%$. For its part, the highest efficiency of bacteria removal is reached in $6 \mathrm{~h}$ with $50.21 \%$. In the absorption process, high turbidity removal efficiency was obtained with a value of $95.24 \%$ in $12 \mathrm{~h}$. In turn, the best results of removal of TSS and TVS were observed at $24 \mathrm{~h}$ with 34.13 and 75.47, respectively. Regarding the nitrogen nutrients, the highest removal efficiency was obtained with ammonium in $12 \mathrm{~h}$ with $39.36 \%$. For the phosphate a value of $87.31 \%$ was recorded in the same time. It is concluded that sedimentation, filtration and absorption processes result in a potential tool for the treatment of effluents. The treatments applied are especially efficient in the reduction of turbidity and phosphates in all times of hydraulic retention, which consequently produces an improvement in the quality of wastewater generated from the production process of $S$. Ialandi.
\end{abstract}

Key words: Wastewater, removal nutrient, sedimentation, filtration, absorption

Resumen.- A escala de laboratorio se evaluó la eficiencia de remoción de material particulado y nutrientes disueltos contenidos en el efluente generado en la producción del pez Seriola lalandi. Fueron aplicados procesos de sedimentación, filtración por la ostra Crassostrea gigas y absorción por la macroalga Gracilaria chilensis en tiempos de retención hidráulica (TRH) de 6, 12 y 24 h. Se utilizaron tanques cilindro cónico de $150 \mathrm{~L}$ de capacidad para el proceso de sedimentación. En los procesos de filtración y absorción se emplearon tanque cilindro cónico de acrílico con $50 \mathrm{~L}$ de capacidad. Todos los tratamientos fueron en triplicado, excepto el proceso de sedimentación que empleó un único tanque para cada tratamiento. La mayor remoción de la turbidez se registró a las $12 \mathrm{~h}$ con un valor de $73,44 \%$. La remoción de los sólidos suspendidos totales (SST) y sólidos volátiles totales (SVT) alcanza la mayor eficiencia a las 24 h con 13,17 y 49,06\%, respectivamente. La remoción de los nutrientes no presenta una alta eficiencia, no obstante, el fosfato en $12 \mathrm{~h}$ se redujo en $82,23 \%$. En el proceso de filtración, la turbidez presenta una alta eficiencia de remoción en todos los TRH evaluados, siendo el mejor resultado 92,57\% en $6 \mathrm{~h}$. Para los SST y SVT, los resultados de remoción fueron 29,94 y $75,4 \%$, respectivamente. En este proceso la reducción de los nutrientes nitrogenados fue muy baja, incluso con valores negativos. No obstante, para el fosfato la mayor remoción fue registrada en $12 \mathrm{~h}$ con 88,32\%. Por su parte, la mayor eficiencia de remoción de bacterias se alcanza en $6 \mathrm{~h}$ con 50,21\%. En el proceso de absorción, fue obtenida una alta eficiencia de remoción de la turbidez con un valor de $95,24 \%$ en $12 \mathrm{~h}$. A su vez, los mejores resultados de remoción de SST y SVT fueron observados a las 24 h con 34,13 y 75,47, respectivamente. En cuanto a los nutrientes nitrogenados, la mayor eficiencia de remoción fue obtenida con el amonio en $12 \mathrm{~h}$ con $39,36 \%$. Para el fosfato se registró un valor de $87,31 \%$ en el mismo tiempo. Se concluye que los procesos de sedimentación, filtración y absorción, resultan en una potencial herramienta para el tratamiento de los efluentes. Los tratamientos aplicados son especialmente eficientes en la reducción de la turbidez y fosfatos en todos tiempos de retención hidráulica, lo que consecuentemente produce un mejoramiento de la calidad de las aguas residuales que se generan a partir del proceso de producción de $S$. lalandi.

Palabras clave: Aguas residuales, remoción de nutrientes, sedimentación, filtración, absorción 


\section{INTRODUCCIÓN}

La acuicultura como muchas actividades humanas produce residuos, que si no se manejan adecuadamente generan un impacto sobre el ambiente (Fuentes 2014). Entre los mayores impactos ambientales que genera la acuicultura, está la descarga directa de los efluentes en aguas costeras y continentales (Chopin et al. 2001). Dentro de esta realidad, el cultivo de peces y camarones son los que más impacto provocan en el ambiente, debido a las características de los efluentes generados en el proceso productivo (PaniaguaMichel \& García 2003). Entre los contaminantes más significativos se encuentran la liberación de excretas, los medicamentos, los productos nitrogenados liberados por los animales y la descomposición del alimento no consumido (Pardo et al. 2006), siendo este último el causante principal del deterioro de la calidad de agua.

Debido a esta problemática, es que surge a nivel internacional el interés en el desarrollo de sistemas de tratamiento de las aguas residuales de la acuicultura (Chopin \& Yarish 1998). Por lo tanto, se deben generar tratamientos eficaces y de bajo costo para obtener una acuicultura sostenible (Naylor et al. 2000). En la última década ha habido un énfasis en desarrollar un enfoque sostenible para la acuicultura y la realización de cultivos en diferentes niveles tróficos son la base para una acuicultura amigable con el ambiente (Neori et al. 2004).

Mediante la integración de distintas especies en cultivo, los desechos de un organismo pueden ser utilizados como recursos alimenticios para el otro. Tal enfoque proporciona una biorremediación del ambiente, creando un beneficio mutuo para las especies que se integran en el cultivo, además existe una diversificación económica mediante la producción de dos productos rentables (Chopin et al. 2001).

Del análisis, surgen alternativas que se utilizan para minimizar los impactos generados por los efluentes de la acuicultura, uso de estanques de sedimentación (TeichertCoddington et al. 1999, Jones et al. 2001, Jackson et al. 2003), absorción de nutrientes por macroalgas (Troell et al. 1997, 1999; Chopin et al. 1999, Neori et al. 2004, Zhou et al. 2006, Buschmann et al. 2008, Paul \& Nys 2008), utilización de microalgas (Hernández \& Labbe 2014, Ramos \& Pizarro 2018) filtración mediante moluscos (Gifford et al. 2004, Ramos et al. 2008) y tratamientos integrados de sedimentación, filtración por moluscos y/o absorción por macroalgas (Jones et al. 2001, Ramos et al. 2010).

Según Teichert-Coddington et al. (1999), los estanques de sedimentación pueden reducir hasta un $61 \%$ los sólidos sedimentables, $40 \%$ los sólidos suspendidos, $12 \%$ la DBO, $7 \%$ el nitrógeno total y $14 \%$ el fósforo total, con no más de $6 \mathrm{~h}$ de tiempo de residencia.

Por su parte, el uso de moluscos filtradores puede ser una alternativa eficiente y económicamente viable para mejorar la calidad de agua de los efluentes de la acuicultura, considerando su capacidad de remoción de fitoplancton, zooplancton, bacterias y microalgas. Al respecto, las ostras pueden ser usadas como biofiltro. Ramos et al. (2008) confirman que Crassostrea rhizophorae es capaz de reducir en forma eficiente la turbidez, los sólidos suspendidos totales, los sólidos volátiles totales, la clorofila $a$ y la $\mathrm{DBO}_{5}$ presente en el agua efluente de cultivo de camarón.

De igual forma, la utilización de macroalgas es una alternativa viable debido a su capacidad de absorber nutrientes disueltos en el agua y utilizarlos para crear nueva biomasa. El uso de algas es la base para el desarrollo de la acuicultura sostenible (Neori et al. 2004). La integración de las algas con peces y camarones, es un medio para la reutilización de los nutrientes disueltos de los efluentes (Troell et al. 1999). Estudios han demostrado que es posible cultivar algas usando las aguas residuales de la acuicultura (Chopin et al. 1999). Especies del género Ulva, Porphyra y Gracilaria han demostrado su eficacia para reducir la carga de nutrientes en los efluentes y ayudar a mantener la calidad del agua (Neori et al. 2004). El género Gracilaria es un candidato atractivo debido a su capacidad para remover nutrientes y a su vez generar productos segundarios de alto valor comercial (Chopin et al. 2001, Neori et al. 2004).

Considerando los antecedentes anteriores, el objetivo de este estudio fue determinar una tecnología de bajo costo y amigable con el ambiente, en el tratamiento de aguas residuales generadas en el cultivo Seriola lalandi. De este modo, se evaluaron procesos de sedimentación, filtración por Crassostrea gigas y absorción por Gracilaria chilensis en distintos tiempos de retención hidráulica (TRH) del efluente.

\section{MATERIALES Y MÉTODOS}

El experimento se realizó en el Laboratorio Integrado de Estudios de Acuicultura (LIEA) del Departamento de Ciencias Acuáticas y Ambientales de la Facultad de Ciencias del Mar y de Recursos Biológicos de la Universidad de Antofagasta.

Las ostras adultas de Crassostrea gigas utilizadas durante el experimento provenían del cultivo de la Empresa Cultivos Marinos Tongoy, Región de Coquimbo. Respecto de Gracilaria chilensis fue recolectada mediante buceo apnea en el submareal de la Caleta Bolsico en el sector Isla Santa María, Región de Antofagasta (2328’31,64”S; 70³6’31,62”O).

El efluente utilizado durante el estudio fue obtenido de reproductores de Seriola lalandi, mantenidos en un tanque de $125 \mathrm{~m}^{3}$ que contenía 21 reproductores de aproximadamente $12 \mathrm{~kg}$ de peso, los cuales eran alimentados diariamente ad libitum con una dieta comercial para salmón modificada para los requerimientos de la especie. El tanque con los reproductores mantenía un flujo abierto de agua, lo 
que determina aproximadamente una tasa de recambio diario del 100\% del volumen total. Con el propósito de recolectar agua de fondo del estanque, se simuló un recambio, recolectando desde el pozo de drenaje aproximadamente 600 $\mathrm{L}$ de efluente bruto. El agua recolectada, fue bombeada a los tanques de sedimentación y previo al inicio del experimento se procedió a una agitación mecánica del efluente para evitar la sedimentación anticipada de partículas. Una vez homogenizado el efluente bruto en los tanques de sedimentación, se recolectaron muestras en triplicado de 500 $\mathrm{mL}$ para realizar los análisis de los parámetros de calidad de agua del efluente bruto.

\section{SEDIMENTACIÓN}

En el proceso de sedimentación se utilizaron 3 tanques cilindro cónico con una capacidad de $150 \mathrm{~L}$, los cuales fueron abastecidos con $120 \mathrm{~L}$ del efluente bruto del estanque de cultivo de dorado. Se evaluaron 3 tiempos de retención hidráulica del efluente (TRH) es decir de 6, 12 y 24 h. Al finalizar cada tiempo experimental se obtuvieron muestras de agua en triplicado de $500 \mathrm{~mL}$, para el análisis de los parámetros de calidad de agua. El efluente resultante de cada tratamiento fue transferido a las correspondientes unidades experimentales del proceso de filtración.

\section{FILTRACIÓN POR MOLUSCOS}

En el proceso de filtración se emplearon los mismos TRH $(6,12$ y 24 h) de la etapa anterior. Para cada tratamiento, fueron utilizados 3 tanques cilindro cónicos de acrílico transparente con una capacidad total de $50 \mathrm{~L}$, los cuales fueron abastecidos con $30 \mathrm{~L}$ del efluente sobrenadante del proceso de sedimentación correspondientes a los
TRH evaluado en la etapa anterior. Los tanques estaban provistos con una plataforma plástica interna para evitar la aglomeración de las ostras en el fondo cónico del estanque. Cada unidad experimental contenía aproximadamente $1.200 \mathrm{~g}$ de ostras adultas de la especie Crassostrea gigas, equivalentes a 7 - 8 individuos, excepto el tanque control que contenía conchas de ostras sin animal. Todas las unidades estaban implementadas con una línea individual de aire para mantener los niveles de oxígeno conforme los requerimientos de la especie, además de mantener el material particulado en suspensión. Terminado el proceso de filtración, desde cada tratamiento se recolectaron muestras de agua en duplicado de $500 \mathrm{~mL}$, para la realización de los análisis físicos y químicos. Posteriormente, el agua sobrenadante fue transferida a los correspondientes tanques para el proceso de absorción.

\section{AbSORCión POR MACROALGAS}

En esta etapa, se utilizaron tanques de las mismas características de la etapa de filtración, cada TRH fue evaluado en triplicado, conteniendo $500 \mathrm{~g}$ peso húmedo de Gracilaria chilensis, con el propósito de mantener la relación entre biomasa de alga y volumen de efluente (Ramos et al. 2008). Se empleó un tanque control, que contenía únicamente el efluente. Cada unidad experimental fue provista de una línea de aire, para mantener el sistema oxigenado y las macroalgas en suspensión. Del mismo modo que en los procesos anteriores, se obtuvieron muestras en duplicado de $500 \mathrm{~mL}$ de cada tratamiento para realizar los análisis de calidad de agua. El diseño experimental utilizado con los procesos de sedimentación, filtración y absorción en los tres tiempos de retención hidráulica se muestra en la Figura 1.

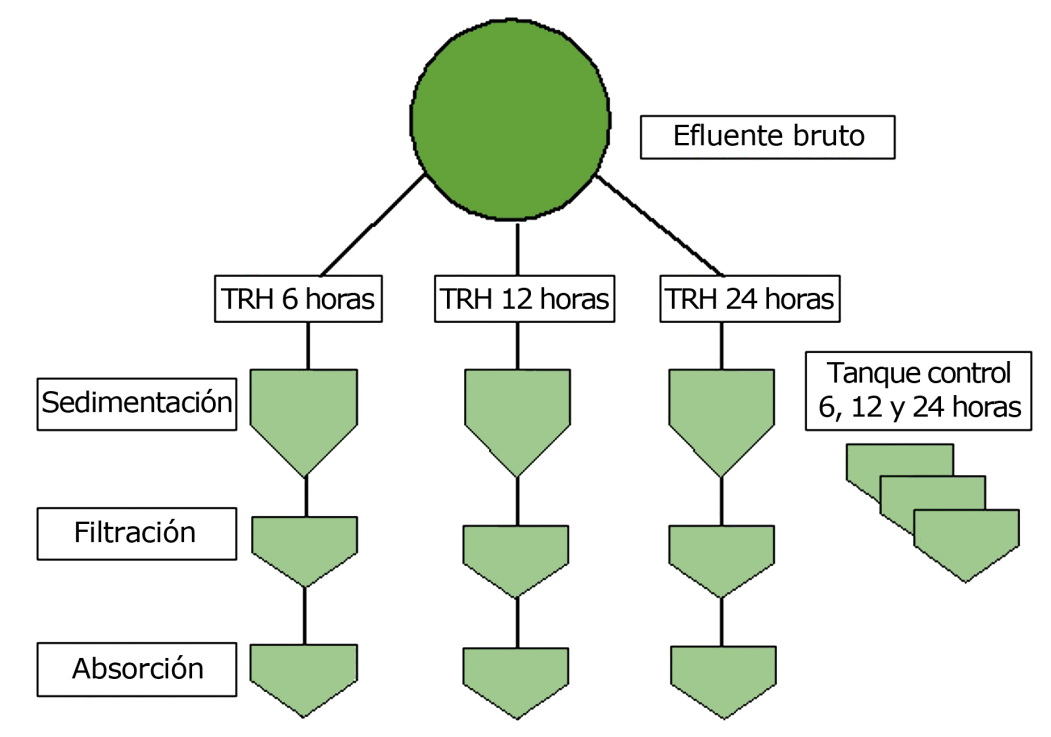

Figura 1. Diseño experimental con la disposición de los tanques en los procesos de sedimentación, filtración y absorción para los tres tiempos de retención hidráulica (TRH) / Experimental design with the arrangement of the tanks in the processes of sedimentation, filtration and absorption for the three hydraulic retention times (HRT) 


\section{DETERMINACIÓN DE PARÁMETROS FÍSICOS Y QUÍMICOS}

La temperatura $\left(\mathrm{T}^{\circ}\right)$, salinidad, $\mathrm{pH}$ y oxígeno disuelto (OD) fueron medidos con un equipo multiparámetro HANNA ${ }^{\circledR}$ Instruments modelo HI9828. La turbidez fue determinada mediante un equipo turbidímetro HANNA ${ }^{\circledR}$ I98703 y los valores expresados en unidades nefelométricas (NTU). En la determinación de los nutrientes, el amonio $\left(\mathrm{NH}_{4}^{+}\right)$, nitrito $\left(\mathrm{NO}_{2}^{-}\right)$, nitrato $\left(\mathrm{NO}_{3}^{-}\right)$y fosfato $\left(\mathrm{PO}_{4}^{-}\right)$se utilizó el espectrofotómetro Spectroquant NOVA 60 (Merck $\left.{ }^{\circledR}\right)$. Por su parte, los sólidos suspendidos totales y sólidos volátiles totales fueron determinados a partir de lo establecido en APHA (1998). La determinación de bacterias totales expresado como unidades formadoras de colonias (UFC) se realizó mediante el método de siembra por extensión, realizando el contaje de las UFC presentes.

Para evaluar la eficiencia de remoción de nutrientes se utilizó la relación propuesta por Paniagua-Michel \& García (2003):

$$
\operatorname{ER}(\%)=\left[\frac{C a-C e}{C a}\right] \cdot 100
$$

donde, $\mathrm{Ca}=$ Concentración del nutriente en el afluente y $\mathrm{Ce}=$ Concentración del nutriente en el efluente.

Los resultados obtenidos fueron analizados empleando análisis de varianza (ANOVA). Previo a la aplicación del ANOVA, los datos fueron transformados a sus equivalentes arco seno, además se determinó la normalidad de los datos y homogeneidad de la varianza mediante la aplicación del test de Bartlet y Kolmogorov-Smirnov, respectivamente. Cuando fueron determinadas diferencias significativas en el análisis de varianza fue aplicado el test de Tukey $(P<$ $0,05)$. En todos los análisis estadísticos se utilizó el software Assistat versión 7.5 beta (Silva \& Azevedo 2006).

\section{RESUltados}

En general, la temperatura, salinidad, $\mathrm{pH}$ y $\mathrm{OD}$ se mantuvieron estables en todos los procesos y en los distintos tiempos de retención hidráulica (Tabla 1). No obstante, el OD en el proceso de sedimentación presentó valores inferiores a los registrados en los otros procesos, independiente del tiempo utilizado.

\section{SEDimentación}

Los valores de remoción de la turbidez presentaron diferencias significativas entre los distintos TRH $(P<0,05)$. La mayor eficiencia de remoción se registró en el tratamiento de $12 \mathrm{~h}$ con un valor de $73,44 \%$, los tratamientos de 6 y 24 h alcanzaron valores de 41,17 y $63,0 \%$, respectivamente (Tabla 2).

En este proceso, los sólidos suspendidos totales (SST) no presentaron diferencias significativas $(P>0,05)$ entre tratamientos. El mayor porcentaje de remoción se presentó en $24 \mathrm{~h}$ con un valor de $13,17 \%$, en 6 y $12 \mathrm{~h}$ registraron valores de 10,76 y 8,93\%, respectivamente. Los sólidos volátiles totales (SVT) en el tratamiento de $24 \mathrm{~h}$ presentaron

Tabla 1. Valores promedios $(n=3)$ de temperatura, salinidad, OD y pH del agua en el efluente bruto, sedimentación, filtración y absorción en los distintos tiempos de retención hidráulica / Average values $(n=3)$ of temperature, salinity, OD and $\mathrm{pH}$ of the water in the raw effluent, sedimentation, filtration and absorption in the different hydraulic retention times

\begin{tabular}{ccccc}
\hline Tratamientos & $\begin{array}{c}\text { Temperatura } \\
\left({ }^{\circ} \mathrm{C}\right)\end{array}$ & Salinidad & $\begin{array}{c}\mathrm{OD}^{-1} \\
\left(\mathrm{mg} \mathrm{O}_{2} \mathrm{~L}^{-1}\right)\end{array}$ & $\mathrm{pH}$ \\
\hline 6 horas de TRH & & & & \\
Efluente bruto & $16,39 \pm 0,11$ & $34,34 \pm 0,06$ & $7,62 \pm 0,19$ & $7,48 \pm 0,33$ \\
Sedimentación & $16,41 \pm 0,05$ & $34,27 \pm 0,03$ & $6,22 \pm 0,18$ & $8,09 \pm 0,10$ \\
Filtración & $15,65 \pm 0,16$ & $34,07 \pm 0,36$ & $7,48 \pm 0,03$ & $8,13 \pm 0,24$ \\
Absorción & $16,09 \pm 0,15$ & $34,42 \pm 0,20$ & $7,44 \pm 0,15$ & $8,57 \pm 0,17$ \\
12 horas de TRH & & & & \\
Efluente bruto & $16,80 \pm 0,05$ & $34,28 \pm 0,07$ & $7,59 \pm 0,27$ & $7,71 \pm 0,03$ \\
Sedimentación & $17,36 \pm 0,05$ & $34,40 \pm 0,12$ & $6,19 \pm 0,06$ & $7,63 \pm 0,03$ \\
Filtración & $15,95 \pm 0,11$ & $34,51 \pm 0,22$ & $7,25 \pm 0,41$ & $8,17 \pm 0,12$ \\
Absorción & $17,21 \pm 0,13$ & $34,67 \pm 0,02$ & $7,07 \pm 0,12$ & $8,04 \pm 0,09$ \\
24 horas de TRH & & & & \\
Efluente bruto & $17,10 \pm 0,15$ & $34,35 \pm 0,32$ & $7,62 \pm 0,19$ & $7,74 \pm 0,03$ \\
Sedimentación & $17,37 \pm 0,05$ & $34,23 \pm 0,08$ & $6,22 \pm 0,18$ & $7,80 \pm 0,01$ \\
Filtración & $16,82 \pm 0,11$ & $34,58 \pm 0,08$ & $7,48 \pm 0,03$ & $8,08 \pm 0,01$ \\
Absorción & $17,32 \pm 0,14$ & $34,84 \pm 0,07$ & $7,44 \pm 0,15$ & $8,66 \pm 0,07$ \\
\hline
\end{tabular}

TRH: tiempos de retención hidráulica 
Tabla 2. Valores promedios $(n=3)$ durante el proceso de sedimentación en los tratamientos de 6, 12 y 24 h de TRH

/ Mean values $(n=3)$ during the sedimentation process in the treatments of 6,12 and 24 h of HRT

\begin{tabular}{|c|c|c|c|c|c|c|c|c|}
\hline Tratamientos & $\begin{array}{c}\text { Turbidez } \\
\text { (NTU) }\end{array}$ & $\begin{array}{c}\mathrm{SST} \\
\left(\mathrm{mgL}^{-1}\right)\end{array}$ & $\begin{array}{c}\text { SVT } \\
\left(\mathrm{mgL}^{-1}\right)\end{array}$ & $\begin{array}{c}\mathrm{NH}_{4}^{+} \\
\left(\mathrm{mgL}^{-1}\right)\end{array}$ & $\begin{array}{c}\mathrm{NO}_{2}^{-} \\
\left(\mathrm{mgL}^{-1}\right)\end{array}$ & $\begin{array}{c}\mathrm{NO}_{3}^{-} \\
\left(\mathrm{mgL}^{-1}\right)\end{array}$ & $\begin{array}{c}\mathrm{PO}_{4}^{-} \\
\left(\mathrm{mgL}^{-1}\right)\end{array}$ & $\begin{array}{l}\text { Bacterias } \\
\left(\mathrm{UFCmL}^{-1}\right)\end{array}$ \\
\hline \multicolumn{9}{|l|}{6 horas de TRH } \\
\hline Efluente bruto & $10,36 \pm 0,73$ & $114,67 \pm 1,15$ & $53,33 \pm 4,04$ & $0,41 \pm 0,01$ & $0,55 \pm 0,01$ & $0,41 \pm 0,00$ & $2,03 \pm 0,06$ & $23.500 \pm 3.000$ \\
\hline Sedimentación & $6,10 \pm 0,04$ & $102,33 \pm 1,53$ & $46,5 \pm 0,71$ & $0,39 \pm 0,00$ & $0,54 \pm 0,02$ & $0,23 \pm 0,00$ & $1,13 \pm 0,15$ & $58.700 \pm 7.200$ \\
\hline \% remoción & $41,17 \mathrm{c}$ & $10,76 \mathrm{a}$ & $12,81 b$ & $4,10 \mathrm{~b}$ & 2,42 & $44,72 \mathrm{a}$ & $44,26 b$ & $-149,79$ \\
\hline \multicolumn{9}{|l|}{12 horas de TRH } \\
\hline Efluente bruto & $34,37 \pm 1,67$ & $130,67 \pm 1,53$ & $80,33 \pm 1,53$ & $0,63 \pm 0,02$ & $0,45 \pm 0,02$ & $0,36 \pm 0,01$ & $6,57 \pm 0,21$ & $18.300 \pm 1.300$ \\
\hline Sedimentación & $9,13 \pm 0,17$ & $119 \pm 1,00$ & $75,33 \pm 0,58$ & $0,60 \pm 0,02$ & $0,42 \pm 0,02$ & $0,35 \pm 0,00$ & $1,17 \pm 0,12$ & $17.100 \pm 3.800$ \\
\hline \% remoción & $73,44 a$ & $8,93 a$ & $6,22 b$ & $4,79 b$ & 5,97 & $3,67 b$ & $82,23 a$ & 6,56 \\
\hline \multicolumn{9}{|l|}{24 horas de TRH } \\
\hline \% remoción & $63,16 b$ & $13,17 \mathrm{a}$ & $49,06 \mathrm{a}$ & $30,67 a$ & $-19,10$ & $-7,21$ & $50,00 \mathrm{~b}$ & $-21,26$ \\
\hline
\end{tabular}

TRH: tiempos de retención hidráulica. Letras diferentes en la misma columna muestran diferencias significativas $(P<0,05)$

la mejor eficiencia de remoción con un $49,06 \%$, mostrando diferencias significativas $(P<0,05)$ respecto de los otros tiempos utilizados. Los tratamientos de 6 y $12 \mathrm{~h}$ no presentaron diferencias significativas $(P>0,05)$ entre sí, alcanzando valores de remoción de 12,81 y $6,22 \%$, respectivamente (Tabla 2 ).

La mayor eficiencia en la remoción de amonio se observó en el tratamiento de $24 \mathrm{~h}$ con $30,67 \%$, presentando diferencias significativas $(P<0,05)$ respecto de los tratamientos de 6 y $12 \mathrm{~h}$, los cuales no mostraron diferencias significativas $(P>0,05)$ entre sí, con valores de 4,10 y $4,79 \%$ respectivamente, en relación con el efluente bruto. Por su parte, el nitrito se incrementó en el tratamiento de $24 \mathrm{~h}$ registrando valores negativos de $-19,10 \%$, no siendo considerado en el análisis estadístico, en cambio, en el tratamiento de $12 \mathrm{~h}$ se constató el mejor resultado de remoción con un $5,97 \%$, por su parte, el tratamiento de $6 \mathrm{~h}$ registró un porcentaje de remoción de 2,42\%. La remoción de nitrato presentó diferencias significativas $(P$ $<0,05)$ entre los tratamientos de $6(44,72 \%)$ y $12(3,67 \%)$ h, en relación al efluente bruto (Tabla 2).

La mayor remoción de fósforo se logró en el tratamiento de $12 \mathrm{~h}$ con un porcentaje de $82,23 \%$ presentando diferencias significativas $(P<0,05)$ en relación a los demás tratamientos. Entre 6 y 24 h no se observaron diferencias significativas $(P>0,05)$ con valores de 44,26 y $50,0 \%$, respectivamente, en relación al efluente bruto (Tabla 2).

La remoción de bacterias totales en el tratamiento de $12 \mathrm{~h}$ registró un valor de $6,56 \%$, sin embargo, en los tratamientos de 6 y 24 h se registró un aumento en la concentración de bacterias, con valores de eficiencia negativos de 149,79 y $21,26 \%$ de incremento, respectivamente.

\section{FiLTRACIÓN}

Calculada la eficiencia de remoción de la turbidez, respecto del efluente bruto, se observó que el tratamiento de 12 h alcanzó el mejor resultado con un valor de 92,53\% presentando diferencias significativas $(P<0,05)$ respecto a los tratamientos de 6 y $24 \mathrm{~h}$, cuyos valores fueron de 77,03 y $81,24 \%$, respectivamente (Tabla 3 ).

En cuanto a los SST, los mejores resultados de remoción comparado con el efluente bruto, se observaron en el tratamiento de 24 h con $29,94 \%$ presentando diferencias significativas $(P<0,05)$ con los otros dos tratamientos (Tabla 3).

En los SVT se observó que la mayor eficiencia de remoción, en relación al efluente bruto, se registró en el tratamiento de $24 \mathrm{~h}$ con $75,4 \%$, presentando diferencias significativas $(P<0,05)$ con los otros tiempos experimentales, que removieron 14,38 y $24,90 \%$ a las 6 y $12 \mathrm{~h}$, respectivamente, no observándose diferencias significativas $(P>0,05)$ entre sí.

En cuanto a la remoción de amonio, se observó un incremento en el tratamiento de $6 \mathrm{~h}$, alcanzando valores negativos de $-10,66 \%$ comparado con la unidad control. Al contrario, los tratamientos de 12 y $24 \mathrm{~h}$ presentaron valores de remoción de 12,23 y 16,00\%, respectivamente. La mayor disminución en la concentración de amonio en relación al efluente bruto se registró en 12 h, el cual presentó un valor de $9,04 \%$, sin diferencias significativas $(P$ $>0,05)$ con el tratamiento de $6 \mathrm{~h}(6,56 \%)$. En el tratamiento de $24 \mathrm{~h}$ se registró un aumento en la concentración de amonio alcanzado un valor negativo de $-42,67 \%$ (Tabla 3 ). 
Tabla 3. Valores promedios $(n=3)$ durante el proceso de filtración en los tratamientos de 6, 12 y 24 h de TRH / Mean values $(n=3)$ during the filtration process in the treatments of 6,12 and $24 \mathrm{~h}$ of TRH

\begin{tabular}{|c|c|c|c|c|c|c|c|c|}
\hline Tratamientos & $\begin{array}{l}\text { Turbidez } \\
\text { (NTU) }\end{array}$ & $\begin{array}{c}\mathrm{SST}_{\left(\mathrm{mgL}^{-1}\right)}\end{array}$ & $\begin{array}{c}\mathrm{SVT} \\
\left(\mathrm{mgL}^{-1}\right)\end{array}$ & $\begin{array}{c}\mathrm{NH}_{4}^{+} \\
\left(\mathrm{mgL}^{-1}\right)\end{array}$ & $\begin{array}{c}\mathrm{NO}_{2}^{-} \\
\left(\mathrm{mgL}^{-1}\right)\end{array}$ & $\begin{array}{c}\mathrm{NO}_{3}^{-} \\
\left(\mathrm{mgL}^{-1}\right)\end{array}$ & $\begin{array}{c}\mathrm{PO}_{4}^{-} \\
\left(\mathrm{mgL}^{-1}\right)\end{array}$ & $\begin{array}{l}\text { Bacterias } \\
\left(\mathrm{UFCmL}^{-1}\right)\end{array}$ \\
\hline \multicolumn{9}{|l|}{6 horas de TRH } \\
\hline Efluente bruto & $10,36 \pm 0,73$ & $114,67 \pm 1,15$ & $53,33 \pm 4,04$ & $0,41 \pm 0,01$ & $0,55 \pm 0,01$ & $0,41 \pm 0,00$ & $2,03 \pm 0,06$ & $23.500 \pm 3.000$ \\
\hline Control & 3,9 & 98,0 & 51,0 & 0,45 & 0,59 & 0,38 & 0,9 & - \\
\hline Filtración & $2,38 \pm 0,13$ & $98,00 \pm 1,73$ & $45,67 \pm 1,15$ & $0,38 \pm 0,01$ & $0,56 \pm 0,01$ & $0,35 \pm 0,02$ & $0,93 \pm 0,06$ & $11.700 \pm 3.400$ \\
\hline$\%$ remoción control & 62,37 & 14,53 & 4,38 & $-10,66$ & $-7,27$ & 7,32 & 55,74 & -- \\
\hline$\%$ remoción E. bruto & $77,03 \mathrm{~b}$ & $14,53 \mathrm{c}$ & $14,38 b$ & $6,56 a$ & $-1,21$ & $14,63 \mathrm{a}$ & $54,10 \mathrm{~b}$ & 50,21 \\
\hline \multicolumn{9}{|l|}{12 horas de TRH } \\
\hline Efluente bruto & $34,37 \pm 1,67$ & $130,67 \pm 1,53$ & $80,33 \pm 1,53$ & $0,63 \pm 0,02$ & $0,45 \pm 0,02$ & $0,36 \pm 0,01$ & $6,57 \pm 0,21$ & $18.300 \pm 1.300$ \\
\hline Control & 3,33 & 101,0 & 69,0 & 0,55 & 0,45 & 0,34 & 1,2 & - \\
\hline Filtración & $2,55 \pm 0,05$ & $97,33 \pm 1,15$ & $60,33 \pm 1,15$ & $0,57 \pm 0,03$ & $0,44 \pm 0,02$ & $0,35 \pm 0,01$ & $0,77 \pm 0,12$ & $15.500 \pm 500$ \\
\hline$\%$ remoción control & 90,31 & 22,70 & 14,11 & 12,23 & $-0,75$ & 6,42 & 81,73 & -- \\
\hline \% remoción E. bruto & $92,57 \mathrm{a}$ & $25,51 b$ & $24,90 \mathrm{~b}$ & $9,04 \mathrm{a}$ & 1,49 & $2,75 b$ & $88,32 \mathrm{a}$ & 15,30 \\
\hline \multicolumn{9}{|l|}{24 horas de TRH } \\
\hline Efluente bruto & $5,81 \pm 0,07$ & $55,67 \pm 3,06$ & $17,67 \pm 0,58$ & $0,25 \pm 0,01$ & $0,30 \pm 0,01$ & $0,37 \pm 0,02$ & $1,53 \pm 0,21$ & $20.700 \pm 2.500$ \\
\hline Control & 2,31 & 43,0 & 7,0 & 0,21 & 0,37 & 0,37 & 0,7 & -- \\
\hline Filtración & $1,09 \pm 0,26$ & $39,00 \pm 1,00$ & $4,33 \pm 1,15$ & $0,36 \pm 0,04$ & $0,30 \pm 0,01$ & $0,49 \pm 0,03$ & $0,80 \pm 0,00$ & $20.100 \pm 1.500$ \\
\hline$\%$ remoción control & 60,24 & 22,75 & 60,38 & 16,00 & $-24,72$ & 0,00 & 54,35 & -- \\
\hline \% remoción E. bruto & $81,24 b$ & $29,94 a$ & $75,4 a$ & $-42,67$ & 0,00 & $-31,53$ & $47,83 \mathrm{c}$ & 2,90 \\
\hline
\end{tabular}

TRH: tiempos de retención hidráulica. Letras diferentes en la misma columna muestran diferencias significativas $(P<0,05)$

En relación al efluente bruto, el nitrito registró la mayor eficiencia de remoción en $12 \mathrm{~h}$ alcanzando un valor de $1,49 \%$. El tratamiento de 24 h no registró remoción. En el tratamiento de $6 \mathrm{~h}$ se observó un incremento llegando a un valor negativo de $-1,21 \%$ (Tabla 3 ).

En cuanto al nitrato, la eficiencia de remoción en el proceso de filtración presentó valores de 7,32 y 6,42\% para los tratamientos de 6 y $12 \mathrm{~h}$, respectivamente, en relación a la unidad control. No se registró remoción en el tratamiento de $24 \mathrm{~h}$. En relación al efluente bruto, la mayor remoción se alcanzó en 6 h con un valor de $14,63 \%$ presentando diferencias significativas $(P<0,05)$ con el tratamiento de $12 \mathrm{~h}$, el cual registró una remoción de $2,75 \%$. El tratamiento de $24 \mathrm{~h}$ presentó un aumento de concentración de nitrato, alcanzando un valor negativo de $-31,53 \%$ (Tabla 3 ).

En la remoción de fosfato el mejor desempeño se registró en el tratamiento de $12 \mathrm{~h}$ con un valor de $81,73 \%$. Los tratamientos de 6 y 24 h alcanzaron valores de 55,74 y $54,35 \%$ respecto a la unidad control. Comparado con el efluente bruto, el mejor desempeño fue alcanzado en el tratamiento de $12 \mathrm{~h}$ con un valor de $88,32 \%$, presentando diferencias significativas $(P<0,05)$ respecto a los tratamientos de 6 y 24 h cuyos valores fueron de 54,10 y $47,83 \%$, respectivamente (Tabla 3 ).
En cuanto a la remoción de bacterias, se observó en el tratamiento de $6 \mathrm{~h}$ una disminución del 50,21\%, seguido del tratamiento de $12 \mathrm{~h}$ con una remoción del 15,30\% y finalmente el tratamiento de $24 \mathrm{~h}$ con un valor de 2,90\%, comparados con el efluente bruto (Tabla 3 ).

\section{ABSORCIÓN}

Los resultados de la eficiencia de remoción por Gracilaria chilensis para todos los parámetros evaluados en los 3 tiempos de retención hidráulica del efluente, se observan en la Tabla 4.

En relación a la turbidez, el tratamiento de $12 \mathrm{~h}$ fue el que presentó el mejor desempeño con un valor de $93,13 \%$, seguida de los tratamientos de 6 h con $66,52 \%$ y 24 h con $61,96 \%$. en relación a la unidad control. Calculada la eficiencia de remoción respecto del efluente bruto, el tratamiento de 12 h alcanzó un valor de 95,24\%, presentando diferencias significativas $(P<0,05)$ respecto a los tratamientos de 6 y $24 \mathrm{~h}$, cuyos valores fueron de 80,86 y $79,52 \%$, respectivamente.

La remoción de los SST en relación al efluente bruto, registró la mayor reducción de a las 24 h de TRH, presentando diferencias significativas $(P<0,05)$ respecto a los tratamientos de 6 y $12 \mathrm{~h}$, con valores 11,34 y $25,0 \%$, respectivamente. 
Tabla 4. Valores promedios $(n=3)$ de las eficiencias de remoción durante el proceso de absorción en los tiempos de retención hidráulica de 6,12 y $\mathbf{2 4} \mathbf{h}$ / Average values $(\mathrm{n}=3)$ of the removal efficiencies during the absorption process in the hydraulic retention times of 6,12 and $24 \mathrm{~h}$

\begin{tabular}{|c|c|c|c|c|c|c|c|c|}
\hline Tratamientos & $\begin{array}{l}\text { Turbidez } \\
\text { (NTU) }\end{array}$ & $\begin{array}{c}\mathrm{SST} \\
\left(\mathrm{mgL}^{-1}\right)\end{array}$ & $\begin{array}{c}\mathrm{SVT} \\
\left(\mathrm{mgL}^{-1}\right)\end{array}$ & $\begin{array}{c}\mathrm{NH}_{4}^{+} \\
\left(\mathrm{mgL}^{-1}\right)\end{array}$ & $\begin{array}{c}\mathrm{NO}_{2}^{-} \\
\left(\mathrm{mgL}^{-1}\right)\end{array}$ & $\begin{array}{c}\mathrm{NO}_{3}^{-} \\
\left(\mathrm{mgL}^{-1}\right)\end{array}$ & $\begin{array}{c}\mathrm{PO}_{4}^{-} \\
\left(\mathrm{mgL}^{-1}\right)\end{array}$ & $\begin{array}{c}\text { Bacterias } \\
\left(\mathrm{UFCmL}^{-1}\right)\end{array}$ \\
\hline \multicolumn{9}{|l|}{6 horas de TRH } \\
\hline Efluente bruto & $10,36 \pm 0,73$ & $114,67 \pm 1,15$ & $53,33 \pm 4,04$ & $0,41 \pm 0,01$ & $0,55 \pm 0,01$ & $0,41 \pm 0,00$ & $2,03 \pm 0,06$ & $23.500 \pm 3.000$ \\
\hline Control & 3,47 & 101,0 & 39,0 & 0,43 & 0,57 & 0,4 & 0,7 & -- \\
\hline Absorción & $1,98 \pm 0,04$ & $101,67 \pm 1,53$ & $34,67 \pm 0,58$ & $0,32 \pm 0,01$ & $0,55 \pm 0,01$ & $0,36 \pm 0,01$ & $0,93 \pm 0,06$ & $17.800 \pm 4.200$ \\
\hline$\%$ remoción control & 66,52 & 11,92 & 26,88 & $-5,74$ & $-3,64$ & 2,44 & 65,57 & -- \\
\hline \% remoción E. bruto & $80,86 b$ & $11,34 \mathrm{c}$ & $35,00 \mathrm{~b}$ & $21,31 b$ & 0,61 & $11,38 \mathrm{a}$ & $54,10 \mathrm{~b}$ & 24,26 \\
\hline \multicolumn{9}{|l|}{12 horas de TRH } \\
\hline Efluente bruto & $34,37 \pm 1,67$ & $130,67 \pm 1,53$ & $80,33 \pm 1,53$ & $0,63 \pm 0,02$ & $0,45 \pm 0,02$ & $0,36 \pm 0,01$ & $6,57 \pm 0,21$ & $18.300 \pm 1.300$ \\
\hline Control & 2,36 & 105,0 & 74,0 & 0,53 & 0,47 & 0,38 & 0,7 & -- \\
\hline Absorción & $1,64 \pm 0,44$ & $98,00 \pm 1,73$ & $57,00 \pm 1,00$ & $0,38 \pm 0,03$ & $0,43 \pm 0,02$ & $0,36 \pm 0,04$ & $0,83 \pm 0,15$ & $16.000 \pm 3.100$ \\
\hline \% remoción control & 93,13 & 19,64 & 7,88 & 15,43 & $-5,22$ & $-4,59$ & 89,34 & -- \\
\hline$\%$ remoción E. bruto & $95,24 \mathrm{a}$ & $25,00 \mathrm{~b}$ & $29,05 b$ & $39,36 \mathrm{a}$ & 3,73 & 1,83 & $87,31 \mathrm{a}$ & 12,57 \\
\hline \multicolumn{9}{|l|}{24 horas de TRH } \\
\hline Efluente bruto & $5,81 \pm 0,07$ & $55,67 \pm 3,06$ & $17,67 \pm 0,58$ & $0,25 \pm 0,01$ & $0,30 \pm 0,01$ & $0,37 \pm 0,02$ & $1,53 \pm 0,21$ & $20.700 \pm 2.500$ \\
\hline Control & 2,21 & 42,0 & 7,0 & 0,22 & 0,37 & 0,35 & 0,7 & -- \\
\hline Absorción & $1,19 \pm 0,16$ & $36,67 \pm 0,58$ & $4,33 \pm 0,58$ & $0,22 \pm 0,01$ & $0,32 \pm 0,01$ & $0,33 \pm 0,02$ & $0,80 \pm 0,00$ & $18.800 \pm 1.900$ \\
\hline$\%$ remoción control & 61,96 & 24,55 & 60,38 & 12,00 & $-24,72$ & 5,41 & 54,35 & -- \\
\hline$\%$ remoción E. bruto & $79,52 b$ & $34,13 a$ & $75,47 \mathrm{a}$ & $13,33 b$ & $-6,74$ & $9,91 \mathrm{a}$ & $47,83 b$ & 9,18 \\
\hline
\end{tabular}

TRH: tiempos de retención hidráulica. Letras diferentes en la misma columna muestran diferencias significativas $(P<0,05)$

Los mayores resultados de remoción de los SVT, calculados en relación al efluente bruto fue en el tratamiento de 24 h con un $75,47 \%$, siendo estadísticamente diferente $(P<0,05)$ a los otros tratamientos. Los tratamientos de $6 \mathrm{~h}$ con $35,0 \%$ y 12 h con $29,05 \%$ de remoción no presentaron diferencias estadísticas $(P>0,05)$ entre sí.

La remoción de amonio, en relación a la unidad control, presentó valores negativos en el tratamiento con $6 \mathrm{~h}$ de TRH con $-5,74 \%$, contrastando con los tiempos de 12 y 24 $\mathrm{h}$, los cuales registraron una remoción de 15,43 y 12,0\%, respectivamente. En relación al efluente bruto, se observó que el mejor desempeño fue en el tratamiento de $12 \mathrm{~h}$ con un de $39,36 \%$, presentando diferencias significativas $(P<$ $0,05)$ respecto de los otros tiempos, los cuales registraron valores de 21,31 y $13,33 \%$ para 6 y $24 \mathrm{~h}$, respectivamente.

La reducción del nitrito en todos los tratamientos alcanzó valores negativos, es decir aumentó su concentración en relación a la unidad control. El tratamiento de 6 h registró un valor negativo de $-3,64 \%$, mientras que los tratamientos de 12 y $24 \mathrm{~h}$ alcanzaron valores de $-5,22$ y $-24,72 \%$, respectivamente. No obstante, la eficiencia de remoción en relación con el efluente bruto, presentó el mejor desempeño en $12 \mathrm{~h}$ con un valor de $3,73 \%$, seguido de $6 \mathrm{~h}$ con una remoción de $0,61 \%$, En cambio, en el tratamiento de $24 \mathrm{~h}$ presentó un valor negativo de $-6,74 \%$.
La remoción del nitrato en el tratamiento de $12 \mathrm{~h}$ presentó un valor negativo de $-4,59 \%$ a diferencia de los tratamientos con 6 y 24 h que registraron valores de remoción de 2,44 y 5,41\%, respectivamente. En relación con los valores del fluente bruto, se constató que no existen diferencias significativas $(P>0,05)$ entre los tratamientos de 6 y $24 \mathrm{~h}$, presentando valores de 11,38 y 9,91\%, respectivamente.

El mejor desempeño en la remoción de fosfato, en relación con la unidad control, se registró en el tratamiento de $12 \mathrm{~h}$ con un valor de $89,34 \%$, seguido de los tiempos de 6 y 24 h con 65,57 y $54,35 \%$, respectivamente. En relación a los valores del efluente bruto, se estableció que la mayor eficiencia de remoción fue en el tratamiento de $12 \mathrm{~h}$ con $89,34 \%$, el cual presentó diferencias significativas $(P<$ $0,05)$ con los otros tiempos evaluados. Los tratamientos de 6 y 24 h no presentaron diferencias significativas $(P>$ $0,05)$ entre sí, alcanzando valores de remoción de 56,10 y $47,83 \%$, respectivamente

El mejor desempeño en la reducción de bacterias totales, en relación al efluente bruto, se observó en el tratamiento de $6 \mathrm{~h}$ con un valor de $24,26 \%$, luego el tratamiento de 12 $\mathrm{h}$ con $12,57 \%$, finalmente el tratamiento de $24 \mathrm{~h}$ de TRH con $9,18 \%$. 


\section{DisCUSIÓN}

En general, los parámetros físicos y químicos durante los procesos de sedimentación, filtración y absorción se mantuvieron estables en los distintos TRH. No obstante, durante el proceso de sedimentación, se registró una leve disminución en el oxígeno disuelto en todos los tiempos evaluados. Esta disminución del nivel de OD puede asociarse al consumo en procesos de respiración por parte de los microorganismos y a la oxidación de compuestos orgánicos presentes en el agua (Ramos et al. 2008).

De acuerdo con Teichert-Coddington et al. (1999), Jones et al. (2001), Jackson et al. (2003) y Ramos et al. (2008), el proceso de sedimentación reduce efectivamente la turbidez, SST y SVT en efluente de cultivo de camarones marinos. Al respecto, Gac \& Virreira (2008) y Gallardo (2009) utilizando efluentes de piscicultura, demostraron que el proceso de sedimentación es eficiente en la reducción del material orgánico e inorgánico presente en el efluente. Al respecto, Gallardo (2009) estableció una eficiencia de remoción de la turbidez de $61,0 \%$ con un TRH de 6 h. Por su parte, Gac \& Virreira (2008) alcanzaron un valor de $24,77 \%$ de remoción, en el mismo TRH. En el presente estudio, los resultados muestran una eficiencia de remoción de la turbidez de $41,0 \%$ en 6 h de TRH, no obstante, el mayor rendimiento se registró en el tiempo de $12 \mathrm{~h}$, alcanzando $73,1 \%$, por lo tanto, son resultados concordantes con los autores anteriores.

Los mayores resultados de eficiencia de remoción de SST y SVT fueron alcanzados en el tratamiento de $24 \mathrm{~h}$ de TRH. Al respecto, los valores registrados en el estudio de Ramos et al. (2008) para SST y SVT fueron de 35,7 y $36 \%$ en 6 y 24 h de TRH, respectivamente, utilizando aguas residuales del cultivo de camarones peneidos.

El proceso de sedimentación no es un método efectivo para la remoción de amonio (Teichert-Coddintong et al. 1999, Ramos et al. 2008, 2010). En este estudio, el tratamiento con $24 \mathrm{~h}$ de TRH obtuvo la mayor eficiencia de remoción. Esta mayor eficiencia de remoción podría ser explicada por el mayor tiempo de residencia del efluente, disponiendo mayor tiempo para actuar las bacterias nitrificantes. Esto último no fue verificado, por lo tanto, se abre una línea de investigación futura.

Para el caso del nitrito, la mayor eficiencia de remoción fue $5,97 \%$ en $12 \mathrm{~h}$, lo cual difiere con lo obtenido por Gallardo (2009) con $22,75 \%$ en 6 h. En el caso del nitrato, la mayor eficiencia se obtuvo en $6 \mathrm{~h}$ con $44,72 \%$, valor inferior al obtenido por Gac \& Virreira (2008) que alcanzaron una eficiencia de remoción de $55,56 \%$ en el mismo TRH.
Según Jones et al. (2001), Gallardo (2009) y Ramos et al. (2010) establecieron que el proceso de sedimentación no es eficiente para la remoción de fosfato. En ese aspecto, Ramos et al. (2010) obtuvieron una remoción de solo $15 \%$ en $6 \mathrm{~h}$ de TRH. No obstante, en el presente estudio los resultados alcanzados, con un valor de $82,23 \%$ en $12 \mathrm{~h}$, indicarían que este proceso si es eficiente, comparado con el resultado de los autores anteriores. Al respecto, Teichert-Coddintong et al. (1999) indican que la mayor concentración de fósforo total en los estanques de camarones marinos se encuentra en el fondo del estanque, lo que lleva a inferir que ese nutriente presenta una mayor capacidad de precipitación si se compara con otros elementos disueltos.

Durante el proceso de sedimentación, la remoción de bacterias totales fue baja, si se compara con los resultados obtenidos por Ramos et al. (2010), los cuales alcanzaron una eficiencia de remoción de 92,3\% utilizando aguas efluentes provenientes de camaronera.

En relación al proceso de filtración, diversos estudios han confirmado que los moluscos bivalvos son utilizados como biofiltro, debido a su alta capacidad de remoción de fitoplancton y sólidos suspendidos (Jones et al. 2001, Gac \& Virreira 2008, Ramos et al. 2008, 2010). Jones et al. (2001) señalan que la ostra Saccostrea commercialis tiene la capacidad de remover altas concentraciones de fitoplancton, bacterias y otros sólidos suspendidos en la columna de agua. Además, indican que las ostras durante la filtración seleccionan las partículas por tamaño, peso y su composición química. A ese respecto, las ostras pueden filtrar partículas tan pequeñas como $1 \mu \mathrm{m}$, aunque su eficiencia en la eliminación de las partículas pequeñas (1-3 $\mu \mathrm{m})$ es sustancialmente inferior a la de partículas mayores. Debido a esas características, se han utilizado distintas especies de ostras como biofiltro, obteniendo eficientes resultados en las remociones de turbidez, SST y SVT (Ramos et al. 2008, 2010).

En cuanto al amonio, los valores de remoción alcanzados en 12 y 24 h (12,23 y 16\%, respectivamente), si bien es cierto son valores bajos, no pueden ser comparados con los resultados negativos obtenidos, tanto por Jones et al. (2001) como por Ramos et al. (2008) para los mismos TRH. Al respecto, Jones et al. (2001) señalaron que las ostras excretan amonio, aminoácidos, urea, ácido úrico y fosfato, por lo tanto, el obtener valores negativos de remoción de amonio estaría relacionado con la característica amoniotélica de la ostra, lo que haría aumentar los niveles en el agua. 
Por su parte, la eficiencia de remoción de nitritos alcanzó valores negativos en todos los tiempos evaluados. Para el caso de los nitratos, los valores de eficiencia de remoción fueron bastante exiguos en todos los tratamientos. Estos resultados estarían confirmando que el proceso de filtración por moluscos, no es eficiente en la reducción de los productos nitrogenados debido a la característica excretora de la ostra C. gigas.

Según Jones et al. (2001), las ostras pueden asimilar directamente el fosfato de la columna de agua para el metabolismo de los hidratos de carbono, transferencia de energía y la formación de la concha. Además, el autor señala que la mayor parte del fósforo filtrado por la ostra es almacenada, luego es liberado como fosfato y solo el $3 \%$ es absorbido y convertido en biomasa. Es así como, Jones et al. (2001) en el proceso de filtración por ostras lograron reducir en $14 \%$ el fósforo total en relación con la concentración en el efluente. Por su parte, en este estudio, los valores de remoción alcanzados en relación al efluente bruto son bastante superiores en todos los TRH evaluados, lo cual confirma la eficiencia de este proceso en la reducción del fosfato presente en aguas residuales.

La eficiencia de remoción alcanzada por la ostra $C$. gigas en la concentración de bacterias totales fue algo inferior a los valores determinados por Jones et al. (2001) con $68,4 \%$ utilizando 6 horas de residencia del efluente. Por su parte, Ramos et al. (2010) obtuvieron una disminución en la concentración de bacterias del 89,3\%, en relación al efluente bruto. Los resultados del presente estudio pueden ser explicados por la baja concentración de bacterias totales en el efluente utilizado, lo cual haría menos eficiente el proceso. Por otra parte, estos organismos constituyen solo una parte de la alimentación de los moluscos filtradores (Jones et al. 2001).

Diversos autores (Troel et al.1997, Chopin et al. 2001, Jones et al. 2001, Zhou et al. 2006, Gallardo 2009, Ramos et al. 2010) han demostrado en cultivos asociados de moluscos, peces y camarones que la utilización de macroalgas es eficiente en la reducción de nutrientes en la columna de agua.

En este estudio, la remoción de la turbidez en el proceso de absorción por Gracilaria chilensis alcanzó el valor más elevado en el tratamiento de $12 \mathrm{~h}$, lo cual fue superior al obtenido por Gallardo (2009) con 27,06 y $16,57 \%$ utilizando las macroalgas Ulva lactuca y Gracilaria chilensis, respectivamente.

Respecto de la remoción de los SST y SVT, en relación al efluente bruto, los valores más altos se registraron en el tratamiento de $24 \mathrm{~h}$, siendo superiores a los obtenidos por Gallardo (2009) utilizando 6 h de TRH, que alcanzó $21,31 \%$ para los SST y $42,80 \%$ para SVT. Ramos et al. (2010) estimaron en $24,4 \%$ la eficiencia de remoción de los SST y $33,3 \%$ para los SVT, utilizado la macroalga Ulva fasciata en 6 h de TRH para efluentes de camaroneras.
La eficiencia de remoción de amonio por Gracilaria chilensis alcanza su mayor eficiencia en el tiempo de 12 $\mathrm{h}$ de TRH con 39,36\%. Este valor de remoción obtenido es inferior al alcanzado por Ramos et al. (2010) con 49,6\% utilizando Ulva fasciata en 6 h. De igual manera, Ramos et al. (2008) determinaron valores de eficiencia de remoción de amonio de $47,1 \%$ con un tiempo de $6 \mathrm{~h}$ con la misma especie de macroalga. A pesar de las eficiencias inferiores en la remoción de amonio, a los obtenidos por Ramos et al. (2010), estos permiten confirmar el rol de las macroalgas para absorber los nutrientes nitrogenados contendidos en el efluente, y formar carbono mediante procesos de fotosíntesis, minimizando el riesgo de eutrofización del ambiente (Shpigel \& Neori 1996, Jones et al. 2001, Marinho-Soriano et al. 2009). Adicionalmente, las macroalgas pueden asimilar el $90 \%$ del amonio liberado en sistemas de cultivos intensivos de peces (Chopin et al. 2001).

Los valores de remoción de nitrito en todos los tratamientos fueron bajos. Por su parte, el nitrato siguió la misma tendencia. Los resultados anteriores, son contrarios a los obtenidos por Gallardo (2009) quien con un tiempo de $6 \mathrm{~h}$ y utilizando la misma macroalga, registró elevadas remociones de nitrito y nitrato con 76,92 y $68,09 \%$, respectivamente.

Las diferencias en la absorción de nutrientes en macroalgas, estaría explicado porque factores físicos como la luz y el movimiento del agua, así como la edad de la planta y el almacenamiento de nutrientes en los tejidos, pueden determinar diferencias en las eficiencias de remoción de nutrientes (Marinho-Soriano et al. 2009). La relevancia de estos factores, deberían ser abordados en trabajos futuros, especialmente el efecto de la luz en la capacidad de absorción de nutrientes por parte de las macroalgas.

En el proceso de absorción se pudo constatar que la mejor eficiencia de remoción de fosfato por Gracilaria chilensis, comparado con los valores del efluente bruto, se alcanzó durante el tratamiento de $12 \mathrm{~h}$, seguido de los tratamientos de 6 y $24 \mathrm{~h}$. Estos resultados son coincidentes con los valores obtenidos por Marinho-Soriano et al. (2009) y Jones et al. (2001), además concuerda con lo expresado por estos autores que señalan que existe una gran afinidad de esta especie de macroalga con procesos de absorción de fosfato.

La remoción de bacterias en el proceso de absorción alcanzó su mayor eficiencia en el tratamiento de $6 \mathrm{~h}$ de TRH. En todo caso, los resultados obtenidos difieren a los obtenidos por Ramos et al. (2010) quienes alcanzaron valores superiores, $72,3 \%$ de remoción en igual tiempo de retención hidráulica, lo cual puede ser explicado por la baja concentración de bacterias totales (UFC) presentes en el efluente bruto utilizados en todos los tiempos experimentales. 
Finalmente, se puede concluir que el proceso de sedimentación es una importante ayuda en el proceso de mejoramiento de la calidad de las aguas de los efluentes de Seriola lalandi. Del mismo modo, el proceso de filtración resultó ser eficiente en la remoción de la turbidez, sólidos suspendidos totales (SST) y solidos volátiles totales (SVT). En general, la ostra C. gigas fue un buen biofiltro en la remoción de bacterias totales, especialmente en el tratamiento de $6 \mathrm{~h}$ de TRH. Por su parte, el proceso de absorción por la macroalga $G$. chilensis demostró una alta eficiencia de remoción de nutrientes, especialmente fosfato y amonio en el tratamiento de 12 TRH. Por lo anterior, se concluye que los procesos de sedimentación, filtración y absorción resultan en una potencial herramienta para el tratamiento de los efluentes. Los tratamientos aplicados son especialmente eficientes en la reducción de la turbidez y fosfatos en los diferentes tiempos de retención hidráulica, por lo que producen un mejoramiento de la calidad de las aguas residuales que se generan a partir del proceso de producción del pez dorado Seriola lalandi.

\section{LITERATURA CITADA}

APHA. 1998. Standard methods for the examination of water and wasterwater, 1193 pp. American Public Health Association (APHA). Byrd Prepress, Springfield.

Buschmann A, D Varela, M Hernández-González \& P Houvinen. 2008. Opportunities and challenges for the development of an integrated seaweed-based aquaculture activity in Chile: Determining the physiological capabilities of Macrocystis and Gracilaria as biofilters. Journal of Applied Phycology 20: 571-577.

Chopin T \& C Yarish. 1998. Nutrients or not? That is the question in seaweed aquaculture and the answer depends on the type and purpose of the aquaculture. World Aquaculture Magazine 29(4): 31-33.

Chopin T, C Yarish, R Wilkes, E Belyea, S Lu \& A Mathieson. 1999. Developing Porphyra/salmon integrated aquaculture for bioremediation and diversification of the aquaculture industry. Journal of Applied Phycology 11(5): 463-472.

Chopin T, A Buschmann, C Halling, M Troell, N Kautsky, G Neori, A Kraemer, J Zertuche-González, C Yarish \& C Neefus. 2001. Integrating seaweeds into marine aquaculture systems: a key toward sustainability. Journal of Phycology 37(6): 975-980.

Fuentes J. 2014. Evolución del régimen ambiental de la acuicultura en Chile. Revista de Derecho, Pontificia Universidad Católica de Valparaíso 42: 441-477.

Gac F \& J Virreira. 2008. Procesos de sedimentación y filtración por moluscos bivalvos Crassostrea gigas (Lamarck 1819) Choromytilus chorus (Molina 1782) y Argopecten purpuratus (Lamarck 1819) en el tratamiento de aguas residuales de instalaciones de cultivo de dorado Seriola lalandi (Cuvier Valenciennes 1833). Seminario de Título, Ingeniería en Acuicultura, Facultad de Ciencias del Mar y de Recursos Biológicos, Universidad de Antofagasta, Antofagasta, $36 \mathrm{pp}$.
Gallardo S. 2009. Tratamientos de los efluentes de la producción de dorado Seriola lalandi (Valenciennes, 1833) mediante procesos de sedimentación y absorción por macroalgas para la minimización del impacto ambiental. Seminario de Título, Ingeniería en Acuicultura, Facultad de Ciencias del Mar y de Recursos Biológicos, Universidad de Antofagasta, Antofagasta, $42 \mathrm{pp}$.

Gifford S, R Dunstan, W O'Connor, T Roberts \& R Toia. 2004. Pearl aquaculture profitable environmental remediation? Science of the Total Environment 319(1-3): 27-37.

Hernández A \& J Labbe. 2014. Microalgas, cultivo y beneficios. Revista de Biología Marina y Oceanografía 49: 157-173.

Jackson C, N Preston, M Buford \& P Thompson. 2003. Managing the development of sustainable shrimp farming in Australia: the role of sedimentation ponds in treatment of farm discharge water. Aquaculture 226: 23-34.

Jones A, W Dennison \& N Preston. 2001. Integrated treatment of shrimp effluent by sedimentation, oyster filtration and macroalgal absorption: a laboratory scale study. Aquaculture 193: 155-178.

Marinho-Soriano E, S Nunes, M Carneiro \& D Pereira. 2009. Nutrients removal from aquaculture wastewater using the macroalgae Gracilaria birdiae. Biomass \& Bioenergy 33: 327-331.

Naylor RL, RJ Goldburg, JH Primavera, N Kautsky, MC Beveridge, M Clay, J Folke, C Lubchenko, H Mooney \& M Troell. 2000. Effect of aquaculture on world fish supplies. Nature 405: 1017-1024.

Neori A, T Chopin, M Troell, A Buschmann, G Kraemer, C Halling, M Shpigel \& C Yarish. 2004. Integrated aquaculture: rationale, evolution and state of the art emphasizing seaweed biofiltration in modern aquaculture. Aquaculture 231: 361-391.

Paniagua-Michel J \& O García. 2003. Ex-situ bioremediation of shrimp culture effluent using constructed microbial mats. Aquacultural Engineering 28: 131-139.

Pardo S, H Suárez \& E Soriano. 2006. Tratamiento de efluentes: una vía para la acuicultura responsable. Revista: Medicina Veterinaria y Zootecnia (MVZ), Universidad de Córdova 11: 20-29.

Paul N \& R de Nys. 2008. Promise and pitfalls of locally abundant seaweeds as biofilters for integrated aquaculture. Aquaculture 281: 49-55.

Ramos R \& R Pizarro. 2018. Crecimiento y capacidad de biorremediación de Chlorella vulgaris (Trebouxiophycea, Chlorophyta) cultivada en aguas residuales generadas en el cultivo del pez dorado Seriola lalandi (Perciformes: Carangidae). Revista de Biología Marina y Oceanografía 53(1): 75-86.

Ramos R, L Vinatea \& $\mathbf{R}$ da Costa. 2008. Tratamiento de efluentes del cultivo de Litopenaeus vannamei por sedimentación y filtración de la ostra Crassostrea rhizophorae. Latin American Journal of Aquatic Research 36(2): 235-244. 
Ramos R, L Vinatea, J Santos \& R da Costa. 2010. Tratamiento de efluentes del cultivo de Litopenaeus vannamei mediante procesos de sedimentación, filtración y absorción. Latin American Journal of Aquatic Research 38(2): 188-200.

Shpigel M \& A Neori. 1996. The integrated culture of seaweed, abalone, fish and clams in modular intensive Land-based system: I. proportions of size and projected revenues. Aquacultural Engineering 15: 313-326.

Silva A \& A Azevedo. 2006. A new version of the assistant statistical assistance software. In: World Congress on Computers in Agriculture, pp. 393-396. American Society of Agriculture Engineers, Orlando.

Teichert-Coddington D, D Rouse, A Potts \& C Boyd. 1999. Treatment of harvest discharge form intensive shrimp pond by settling. Aquacultural Engineering 19: 147-161.
Troell M, C Halling, A Nilsson, A Buschmann, N Kautsky \& L Kautsky. 1997. Integrated marine cultivation of Gracilaria chilensis (Gracilariales, Rhodophyta) and salmon cages for reduced environmental impact and increased economic output. Aquaculture 156: 45-61.

Troell M, P Rönnbäck, N Kautsky, C Halling \& A Buschmann. 1999. A ecological engineering in aquaculture: use of seaweeds for removing nutrients from intensive aquaculture. Journal of Applied Phycology 11: 89-97.

Zhou Y, H Yang, H Hu, Y Liu, Y Mao, H Zhou, X Xu \& F Zhang. 2006. Bioremediation potential of the macroalga Gracilaria lemaneiformis (Rhodophyta) integrated into fed fish culture in coastal waters of north China. Aquaculture 252(2-4): 264-276.

Recibido el 28 de enero de 2019 y aceptado el 25 de octubre de 2019

Editor: Claudia Bustos D. 